\title{
Tratamientos pregerminativos para Myrceugenia exsucca (Myrtaceae)
}

\author{
Pregermination treatments of Myrceugenia exsucca (Myrtaceae)
}

\author{
Mirtha Latsague Vidal ${ }^{\mathrm{a} *}$, Patricia Sáez Delgado ${ }^{\mathrm{b}}$, Leandra Coronado Ancaten ${ }^{\mathrm{a}}$ \\ *Autor de correspondencia: aniversidad Católica de Temuco, Escuela de Ciencias Ambientales, Facultad de Recursos Naturales, \\ casilla 15-D, tel.: (45) 205409, fax: (45) 211034, Temuco, Chile, mlatsagu@uct.cl \\ bUniversidad de Concepción, Facultad de Ciencias Forestales, Concepción, Chile.
}

\begin{abstract}
SUMMARY
The knowledge of specific germination requirement is important in plants production using seeds. The majority of the native species need of cultural management, among them those related with appropriate conditions to the germination of their seeds. However, available information to develop this methodology in species of Myrtaceae is still conspicuously lacking, as is the case of Myrceugenia exsucca (pitra). Currently, pitra is the most abundant species in the forested wetlands of the Araucanía Region, thus reports of results that contribute to its propagation are important in the conservation of these ecosystems. The objective of the present work was to evaluate the effect of different pregermination treatments (imbibition, cold stratification and gibberellic acid) on viable seeds of $M$. exsucca through tests of viability and germination under laboratory conditions. The seeds were treated with five treatments: soak in distilled water for $24 \mathrm{~h}$; soak in $250 \mathrm{mg} \mathrm{L}^{-1}$ gibberellic acid for $12 \mathrm{~h}$; soak in $250 \mathrm{mg} \mathrm{L}^{-1}$ gibberellic acid during $24 \mathrm{~h}$; cold stratification at $5{ }^{\circ} \mathrm{C}$ during 15 days. Percentage of germination and index of germination velocity were determined. We found $90 \%$ of viability in our experiments. The results showed that the best results were obtained soaking the seeds in distilled water during $24 \mathrm{~h}$ with an average germination percentage of $71 \%$. This information might be required for the management and conservation of $M$. exsucca
\end{abstract}

Key words: germination, stratification, conservation, wetlands.

\section{RESUMEN}

El conocimiento de los tratamientos pregerminativos específicos resulta de interés en la producción de plantas a través de semillas. La mayoría de las especies nativas necesitan de manejos culturales, entre ellos, los relacionados con las condiciones apropiadas para la germinación de sus semillas. Hasta el momento, especificaciones precisas para el desarrollo de estas metodologías en especies de la familia Myrtaceae son escasas, tal es el caso de Myrceugenia exsucca (pitra), especie más abundante en los humedales boscosos de la región de La Araucanía. Así, informar resultados que contribuyan a su propagación por semillas resulta importante en la conservación de este tipo de ecosistemas. El objetivo de este estudio fue evaluar viabilidad y tratamientos pregerminativos en semillas de $M$. exsucca a través de ensayos de germinación en condiciones de laboratorio. Las semillas fueron tratadas con cuatro tratamientos: remojo con agua destilada por $24 \mathrm{~h}$; remojo en ácido giberélico, $250 \mathrm{mg} \mathrm{L}^{-1}$ por $12 \mathrm{~h}$; remojo en ácido giberélico, 250 $\mathrm{mg} \mathrm{L}{ }^{-1}$ por $24 \mathrm{~h}$ y estratificación fría a $5^{\circ} \mathrm{C}$ por 15 días. Para cada tratamiento se utilizaron cinco repeticiones de 25 semillas cada uno. Se determinó porcentaje de germinación e índice de velocidad de germinación. Como resultado de la prueba de tetrazolium, se obtuvo $90 \%$ de viabilidad. El remojo en agua destilada por $24 \mathrm{~h}$ como tratamiento pregerminativo aumenta el porcentaje de germinación, alcanzando $71 \%$. Esta información podría ser requerida para el manejo y conservación de $M$. exsucca.

Palabras clave: germinación, estratificación, conservación, humedales.

\section{INTRODUCCIÓN}

En la región de La Araucanía, el bosque pantanoso de Myrtaceae forma parte de los bosques templados de la depresión central y Cordillera de la Costa, correspondiendo fitosociológicamente al bosque de temo y pitra (Blepharocalyo-Myrceugenietum exsuccae). Actualmente, esta comunidad boscosa se encuentra en diferentes estados de degradación, siendo especialmente afectada por actividades de extracción de leña, tala y roce a fuego, junto a políticas gubernamentales de incentivo a la recuperación de terrenos para la agricultura mediante el drenado de suelos (Hauenstein et al. 2002, Muñoz-Pedreros y Möller 2002). La eliminación de estos ecosistemas significa una pérdida de la biodiversidad del lugar, siendo Myrceugenia exsucca (D. C.) Berg. (pitra) una de las especies más fuertemente afectadas, lo que ha influido en su clasificación de vulnerable (Benoit 
1989). Esta especie, capaz de retoñar vegetativamente (Donoso 2006), es la mejor adaptada a las condiciones restrictivas de su ambiente. Puede crecer con las raíces sumergidas en el agua logrando un mejor desarrollo en los lugares topográficamente más deprimidos, en donde la napa freática es más superficial (Armesto et al. 1995). Su distribución natural comprende desde Aconcagua hasta Chiloé continental (regiones de Coquimbo a Los Lagos), encontrándose también en Argentina.

Frente a la tendencia actual de pérdida de la biodiversidad, la propagación por semilla contribuye no sólo al mantenimiento de la variabilidad, sino también a su incremento, por lo cual es motivo de numerosas investigaciones. Las condiciones más favorables para el proceso de germinación deben ser establecidas para cada especie, por lo que documentar resultados de tratamientos pregerminativos resulta de interés en programas de conservación ex situ (Ulian et al. 2008). Existen reportes respecto de germinación en especies de la familia Myrtaceae (Ramírez et al. 1980, Figueroa 1996, Meza y Bautista 2007, Otegui et al. 2007); con respecto a M. exsucca, Donoso (2006) señala que sin aplicar tratamientos pregerminativos esta especie presenta $50 \%$ de germinación. Considerando los peligros que enfrenta el hábitat de esta especie por el grado de intervención, el objetivo de este estudio fue evaluar la viabilidad y tratamientos pregerminativos en semillas de $M$. exsucca a través de ensayos de germinación en condiciones de laboratorio, con la finalidad de aportar nuevos antecedentes para su germinación.

\section{MÉTODOS}

Para realizar este estudio se recolectaron en septiembre de 2008 frutos en forma manual de $M$. exsucca, de 10 distintos individuos de la misma población dentro del bosque pantanoso del sector Pumalal, a $25 \mathrm{~km}$ de la ciudad de Temuco $\left(38^{\circ} 35^{\prime} 55^{\prime}\right.$ ' O, $72^{\circ} 31$ ' 20" N); la población ocupa una superficie aproximada de 233 ha.

Las bayas se abrieron en forma manual para remover la pulpa. Las semillas extraídas con una pinza de punta fina fueron espolvoreadas con fungicida Ftalimida ${ }^{1}$ y almacenadas durante un mes en bolsas de papel a temperatura ambiente $\left(18 \pm 3{ }^{\circ} \mathrm{C}\right)$ y humedad ambiental de 50-70\%, hasta la aplicación de los tratamientos pregerminativos. Previa aplicación de los tratamientos se determinó viabilidad de las semillas en cinco repeticiones de 25 semillas cada una. Para esto se aplicó la prueba de tetrazolium (solución acuosa al $0,1 \% \mathrm{p} / \mathrm{v}$ ) durante $24 \mathrm{~h}$ a $30^{\circ} \mathrm{C}$. La viabilidad se expresó en porcentaje de semillas vivas respecto del total de la muestra analizada (Steiner et al. 1999).
De las semillas viables se evaluaron cuatro tratamientos pregerminativos más el control sin pretratamiento (T0): remojo con agua destilada por 24 h (T1), remojo en ácido giberélico $250 \mathrm{mg} \mathrm{L}^{-1}$ por $12 \mathrm{~h}$ (T2), remojo en ácido giberélico $250 \mathrm{mg} \mathrm{L}^{-1}$ por $24 \mathrm{~h}$ (T3) y estratificación fría (refrigerada a $5{ }^{\circ} \mathrm{C}$ y $\mathrm{HR} 10 \% \pm 2 \%$ ) durante 15 días (T4). La incubación se efectuó a temperatura ambiente de $15^{\circ} \mathrm{C} \pm 1^{\circ} \mathrm{C}$ y con un fotoperíodo de $12 \mathrm{~h}$ luz.

Los recuentos de germinación se hicieron diariamente a partir del tercer día de establecido el ensayo (momento en el que todos los tratamientos presentaron semillas germinadas) hasta completar 25 días. Posteriormente, no germinaron más semillas. Se consideró como semilla germinada aquella que presentaba emergencia de la radícula (Herranz et al. 2002) sin considerar su longitud. Se evaluó el porcentaje de germinación (PG) y el índice de velocidad de germinación (IVG) (Maguire 1962) [1].

$$
\mathrm{IVG}=\mathrm{P}_{1} / \mathrm{T}_{1}+\mathrm{P}_{2} / \mathrm{T}_{2}+\mathrm{Pn} / \mathrm{Tn}
$$

Donde P: número de semillas germinadas, T: tiempo transcurrido para esa germinación (en días) y 1, 2, n: recuento diario.

El ensayo fue evaluado por medio de un diseño experimental completamente aleatorio y la unidad experimental estuvo compuesta por una muestra de 25 semillas depositadas en una placa Petri de $10 \mathrm{~cm}$ de diámetro, utilizando como sustrato turba. Se realizaron cinco repeticiones por tratamiento. Los porcentajes de germinación se transformaron en arcoseno, posteriormente se realizó un análisis de varianza (ANDEVA) y las diferencias entre tratamientos fueron evaluadas con la prueba de comparaciones múltiples de Tukey $(P<0,05)$. Para procesar los datos se utilizó el programa Statgraphics Plus versión 5.1.

\section{RESULTADOS}

Como resultado de la prueba de tetrazolium se obtuvo $90 \% \pm 7,8 \%$ de viabilidad. Respecto a la germinación, se presentan datos de semillas germinadas sólo hasta el día 25, momento a partir del cual no se registró aumento en la germinación. En los tratamientos T3 y T4 la germinación fue nula. Entre los tratamientos germinados, el remojo con agua destilada por 24 h (T1) presentó mayor germinación que el tratamiento control $(P<0,05)$ (figura 1). Los valores más bajos se obtuvieron en el tratamiento con ácido giberélico por $12 \mathrm{~h}$ (T2). Sin embargo, este tratamiento presentó el valor más alto de velocidad germinativa, presentando diferencias significativas con el tratamiento control y remojo con agua por $24 \mathrm{~h}(P<0,05)$ (cuadro 1$)$. 


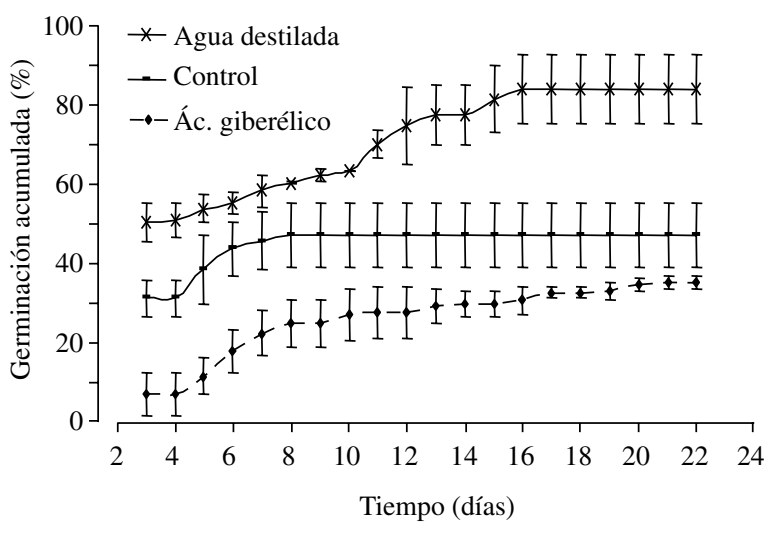

Figura 1. Germinación acumulada (\%) para semillas de Myrceugenia exsucca en función de los tratamientos aplicados. Los resultados de los tratamientos remojo en ácido giberélico por 24 h (T3) y almacenamiento en frío a $5{ }^{\circ} \mathrm{C}$ (T4) no se muestran ya que la germinación fue nula.

Accumulated germination (\%) for seeds of Myrceugenia exsucca based on the applied treatments. The results of the treatment soak in AG3 by $24 \mathrm{~h}(\mathrm{~T} 3)$ and storage in $5{ }^{\circ} \mathrm{C}(\mathrm{T} 4)$ are not since the germination was null.

Cuadro 1. Valores medios \pm desviación estándar de capacidad germinativa y velocidad de germinación en semillas de Myrceugenia exsucca sometidas a distintos tratamientos pregerminativos. Letras distintas indican diferencias significativas $(P<0,05)$.

Average values $( \pm$ SD) of germination capacity $(\%)$ and speed of germination (days) in seeds of Myrceugenia exsucca submissive different pregermination treatments. Different letters indicate significant statistical differences $(P<0.05)$.

\begin{tabular}{lcc}
\hline \multicolumn{1}{c}{ Tratamiento } & $\begin{array}{c}\text { Capacidad } \\
\text { germinativa } \\
(\%)\end{array}$ & $\begin{array}{c}\text { Índice de } \\
\text { velocidad } \\
\text { germinativa (días) }\end{array}$ \\
\hline Control (T0) & $43 \pm 6,0 \mathrm{~b}$ & $4 \pm 0,3 \mathrm{~b}$ \\
Agua destilada (T1) & $71 \pm 4,4 \mathrm{a}$ & $4 \pm 0,2 \mathrm{~b}$ \\
Ácido giberélico (T2) & $25 \pm 4,2 \mathrm{c}$ & $8 \pm 2,0 \mathrm{a}$ \\
\hline
\end{tabular}

\section{DISCUSIÓN}

Los porcentajes de viabilidad obtenidos en esta investigación corroboran lo informado por Hechenleitner et al. (2005), respecto a la alta viabilidad de M. exsucca. El tratamiento de remojo con agua destilada por $24 \mathrm{~h}$ presenta diferencias significativas respecto del control, incrementando los porcentajes de germinación a un $71 \%$, superando el resultado señalado por Donoso (2006), quien informa un $50 \%$ de germinación en semillas de $M$. exsucca sin aplicar pretratamiento. Sin embargo, los porcentajes son menores a los informados por Figueroa et al. (1996) para Myrceugenia planipes en donde se obtuvo un $90 \%$ de germinación. El efecto positivo de la imbibición se compara con lo señalado por Pandey et al. (2000) y Smiderle y Minami (2001), quienes remojaron semillas de Myrtaceae del género Psidium alcanzando porcentajes mayores de germinación que en semillas sin tratar. Por otro lado, Vogel y Doll (1999) reportan entre 70 y $75 \%$ de germinación luego de 24 h de imbibición en agua fría en especies nativas ornamentales. La imbibición como mecanismo de escarificación química favorece la entrada de agua y el intercambio gaseoso necesario para la germinación (Díaz et al. 1994, Otegui et al. 2005). De acuerdo a Razz y Clavero (1996), el tratamiento de imbibición de las semillas es un método sencillo, práctico y económico, que puede ser utilizado para incrementar el porcentaje de germinación en diferentes especies.

$\mathrm{Si}$ bien es conocido que el ácido giberélico incrementa la germinación de numerosas especies, tal como lo señalan varios autores (Araya et al. 2000, Atencio et al. 2003) y es recomendado para el rompimiento de la latencia, los porcentajes de germinación de semillas de $M$. exsucca tratadas con esta hormona no superaron los valores obtenidos en el tratamiento de remojo en agua destilada, como tampoco aquellos obtenidos en el tratamiento control. El efecto del tiempo de inmersión de las semillas en ácido giberélico se compara con los resultados informados por Martínez-Pastur et al. (1994), quienes señalan que en semillas de Nothofagus betuloides (Mirb.) Oerst. sólo el remojo por $12 \mathrm{~h}$ estimula la germinación comparado con el remojo por $24 \mathrm{~h}$. Con los antecedentes actuales de la literatura no se puede dar respuesta a este fenómeno; sin embargo, considerando que la temperatura y la concentración del ácido giberélico fueron las mismas, se puede asumir, aunque no esté reportado, algún tipo de interacción entre el tiempo de inmersión y la actividad de enzimas hidrolíticas responsables de la degradación de reservas de la semilla.

La nula respuesta obtenida en el tratamiento con frío podría deberse a que la exposición al frío provoque deshidratación de la semilla. En este sentido, Ramírez et al. (1980) señalan que las semillas de Myrceugenia spp. presentan un embrión muy expuesto debido a que poseen una testa muy fina y son pobres en endosperma, lo que podría conducir a una deshidratación del embrión.

\section{CONCLUSIONES}

Semillas de $M$. exsucca cosechadas y luego almacenadas durante un mes a temperatura ambiente mantienen un alto porcentaje de viabilidad. Los tratamientos pregerminativos incrementan la germinación de semillas de $M$. exsucca, siendo el remojo de las semillas en agua destilada por $24 \mathrm{~h}$ el que más favorece la emergencia de la radícula.

\section{AGRADECIMIENTOS}

Proyecto FPA (CONAMA) 09-002-2009 y proyecto DGIPUCT 2009-03-04. 


\section{REFERENCIAS}

Araya E, L Gómez, N Hidalgo, R Valverde. 2000. Efecto de la luz y del ácido giberélico sobre la germinación in vitro de Jaul (Alnus acuminata). Agronomía Costarricense 24(1): 75-80.

Armesto J, P León, M K. Arroyo. 1995. Los bosques templados del sur de Chile y Argentina: una isla biogeográfica. In Armesto J, C Villagrán, MK Arroyo eds. Ecología del bosque nativo de Chile. Santiago, Chile. Universitaria. p. 23-28.

Atencio L, R Colmenares, M Ramírez, D Marcano. 2003. Tratamientos pregerminativos en acacia San Francisco (Peltophorum pterocarpum) Fabaceae. Rev. Fac. Agron. 20: 63-71.

Benoit I. 1989. Libro rojo de la flora terrestre de Chile (Primera Parte). Santiago, Chile. Corporación Nacional Forestal CONAF. $157 \mathrm{p}$.

Díaz I, J Viera, A Escobar. 1994. Efecto de diferentes métodos de escarificación sobre la germinación en semillas de Pachecoa venezuelensis Burkart. Agronomía Trop. 45(4): 561-570.

Donoso C. 2006. Las especies arbóreas de los bosques templados de Chile y Argentina. Autoecología. Valdivia, Chile. Marisa Cuneo Ediciones. 678 p.

Figueroa J, J Armesto, J Hernández. 1996. Estrategias de germinación y latencia de semillas en especies del bosque templado de Chiloé, Chile. Revista Chilena de Historia Natural. 69: 243-251

Hauenstein E, M González, F Peña, A Muñoz. 2002. Clasificación y caracterización de la flora y vegetación de los humedales de la costa de Toltén (IX Región de Chile). Gayana Botanica 59(2): 87-100.

Hechenleitner P, M Gardner, P Thomas, C Echeverría, B Escobar, P Brownless, E Martínez. 2005. Plantas amenazadas del Centro Sur de Chile. Distribución, conservación y propagación. Valdivia, Chile. Universidad Austral de Chile-Real Jardín Botánico de Edimburgo. 188 p.

Herranz JM, P Ferrandis, MA Copete, JJ Martínez-Sánchez. 2002. Influencia de la temperatura de incubación sobre la germinación de 23 endemismos vegetales ibéricos o iberoafricanos. Invest. Agrar., Prod. Protecc. Veg. 17(2): 229-245.
Maguire J D. 1962. Speed of germination-aid in selection and evaluation for seedling emergence and vigor. Crop Sci. 2: 176-177.

Martínez Pastur G, M Arena, C Fernández. 1994. Nota sobre la influencia del ácido giberélico y del nitrato de potasio en la germinación de semillas de Nothofagus betuloides (Mirb.) Oerst. Investigación Agraria Sist. Recur. For. 3(1): 83-89.

Meza N, D Bautista. 2007. Morfología de semillas de Guayabo (Psidium guajava L.), germinación y emergencia después del remojo en agua. Rev. Fac. Agron. 24 (1): 265-270.

Muñoz-Pedreros A, Möller P. 2002. Conservación de humedales. Bases para la conservación de humedales de Chile. Valdivia, Chile. Ediciones del Centro de Estudios Agrarios y Ambientales (CEA). 95 p.

Otegui M, MA Pérez, M de Souza Maia. 2005. Efecto de la temperatura y la luz en la germinación de semillas de Paspalum guenoarum. Revista Brasileira de Sementes 27(1): 190-194.

Pandey D, S Gorakh, G Singh. 2000. Effect of seed pretreatment on promotion of germination in Guava (Psidium guva L.) Indian Journal of Agronomy 21(2): 279-281.

Ramírez C, M Romero, O Henríquez. 1980. Estudios de germinación en semillas de mirtáceas chilenas. Bosque 3(2): 106-114.

Razz R, T Clavero. 1996. Métodos de escarificación de semillas de Humboldtiella ferruginea y Leucaena leucocephala. Rev. Fac. Agron. 13: 73-77.

Smiderle O, J Minami. 2001. Emergence and vigor of guava seedlings on different substrates. R S. Brasil. Universidade da Regiao da Campanha. Bage. Revista Científica Rural 2(1):38-45.

Steiner A, M Kruse, H Fuchs. 1999. A re-assessment of the comparison of tetrazolium viability testing and germination testing. Seed Science and Technology 27:59-65.

Ulian T, A Rovere, B Muñoz. 2008. Taller sobre conservación de semillas para la restauración ecológica. Ecosistemas 17(3): 147-148.

Vogel H, U Doll. 1999. Domesticación de diferentes especies nativas ornamentales y medicinales. Talca, Chile. Universidad de Talca. $48 \mathrm{p}$. 